\title{
Heights of meteoric fireballs
}

\author{
observed in 1908 and 1909.
}

The following are the results of some calculations streaks of comparatively durable kind and these streaks have made here of the real paths of a number of fireballs which shown a motion of roo to 120 miles per hour at altitudes have been sufficiently well observed at more than one station. A great number have been seen but few recorded accurately and completely. Several of the meteors have left drifting

from 50 to 60 miles. Some very brilliant Perseids have been seen this year and the shower was well displayed in very clear weather.

\begin{tabular}{|c|c|c|c|c|c|c|c|c|c|c|c|c|}
\hline & Date & & Gr. & M. T. & Mag. & $\begin{array}{l}\text { Height } \\
\text { at be- } \\
\text { ginning }\end{array}$ & $\begin{array}{l}\text { Height } \\
\text { at en- } \\
\text { ding }\end{array}$ & $\begin{array}{l}\text { Length } \\
\text { of } \\
\text { path }\end{array}$ & $\begin{array}{l}\text { Velo- } \\
\text { city pr. } \\
\text { second }\end{array}$ & & $\begin{array}{l}\text { diant } \\
\text { oint }\end{array}$ & \\
\hline & & & & & & miles & miles & miles & miles & 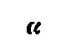 & $\delta$ & \\
\hline 1908 & May & I 7 & & $43^{m}$ & $>q$ & 76 & 28 & 57 & I 5 & $194^{\circ}$ & $+20^{\circ}$ & Coma Berenicid \\
\hline & & 19 & 10 & 20 & $>9$ & 69 & 45 & 142 & 22 & 252 & -20 & $\alpha$ Scorpiid \\
\hline & & 27 & 12 & 28 & $>$ q & $5^{8}$ & 55 & I 20 & I 5 & 76 & +43 & $\alpha$ Aurigid \\
\hline & June & 28 & I I & I 2 & ㅇ & 67 & 45 & 84 & 12 & 237 & -18 & $\theta$ Librid \\
\hline & July & $\mathbf{I}$ & 9 & I 5 & $1 / 2 D$ & 61 & 27 & 105 & 18 & 250 & -20 & a Scorpiid \\
\hline & & 28 & I I & 6 & 5 & 82 & 40 & 50 & 34 & 302 & +23 & $\eta$ Sagittid \\
\hline & Aug. & 2 & 10 & 30 & 24 & 77 & 47 & 57 & $3^{8}$ & $3^{8}$ & +54 & i) Perseid \\
\hline & & 2 & 12 & 7 & 24 & $6 I$ & 49 & 32 & 21 & 337 & - I I & $\delta$ Aquarid \\
\hline & & Io & 10 & 17 & 24 & 72 & 45 & 52 & 29 & 43 & +57 & $\eta$ Perseid \\
\hline & Sept. & 14 & 8 & 48 & $2 \times q$ & 59 & $3^{2}$ & $7 \mathrm{I}$ & - & 6 & +7 & $\gamma$ Pegasid \\
\hline & Oct. & 14 & 9 & 10 & $>9$ & 70 & 18 & 96 & 24 & 42 & +20 & $\varepsilon$ Arietid \\
\hline & & 23 & 12 & 19 & $>Q$ & 73 & 47 & 33 & - & 33 & +16 & c Arietid \\
\hline & Nov. & 16 & I 8 & 32 & $1 / 2 D$ & 85 & $5^{\circ}$ & 41 & - & 150 & +23 & Leonid \\
\hline 1909 & Jan. & I I & 8 & I I & $>9$ & $5^{8}$ & 29 & $5^{2}$ & I 3 & $33^{2}$ & +36 & $\pi$ Pegasid \\
\hline & & 25 & 13 & 25 & 9 & 55 & 42 & 108 & I 5 & 40 & +18 & $\varepsilon$ Arietid \\
\hline & Febr. & 22 & 7 & 33 & $1 / 2 D$ & $5^{6}$ & 50 & I 55 & 25 & 196 & +20 & Coma Berenicid \\
\hline & April & 20 & 9 & 50 & $>q$ & 86 & $5^{6}$ & 90 & - & 270 & +30 & Lyrid \\
\hline & Aug. & 8 & ro & 8 & 24 & 94 & 65 & $5^{8}$ & 48 & $4 \mathrm{I}$ & +57 & $\eta$ Perseid \\
\hline & & 10 & I I & I 5 & 24 & 87 & 52 & 62 & 40 & 43 & +56 & $\eta$ Perseid \\
\hline & & I I & 9 & 57 & 24 & 103 & 48 & I IO & 32 & 43 & $+5^{8}$ & $\eta$ Perseid \\
\hline & & I 2 & 9 & 42 & $3 \times 9$ & 87 & 53 & 68 & 35 & & & $\eta$ Perseid \\
\hline & & 12 & 9 & $5^{I}$ & 24 & 90 & 62 & 58 & 46 & 47 & $+5^{8}$ & \\
\hline & & I 2 & 10 & 19 & 24 & 78 & $5^{2}$ & $5^{2}$ & $3^{\circ}$ & & & $\eta$ Perseid \\
\hline
\end{tabular}

6 observers

Seen in Ireland

Seen at Utrecht and Anvers

Well observed

6 observers

Very brilliant fireball

Left a bright streak

Left a bright streak

Left drifting trail 30 minutes

Lit up the sky

I 7 observers

4 observers

Left a drifting streak for more than 3 hours. More than 200 obs.

Left a drifting streak. Observed in France

Bright streak $\gg$

Lit up the sky and left a brilliant streak

Bright streak

The Perseid fireball of 1909 Aug. $129^{\mathrm{h}} 42^{\mathrm{m}}$ was seen by many observers in London and other towns of England. As viewed by me at Bristol it gave a brilliant flash and left a streak for 45 seconds.

Bristol, I909 Aug. I 9.

W. F. Denning.

\section{Über einen Nebelhaufen in Cetus.}

Gelegentlich der Untersuchung von Platten aus der Umgegend von $\alpha$ Ceti stieß ich auf eine Stelle, wo sich zahlreiche kleine Nebelflecken auf engen Raum zusammendrängen. Dieser Teil des Himmels ist im allgemeinen nebelarm, so daß für das Bruce-Teleskop nur etwa 3-5 Nebelflecken auf den Quadratgrad kommen. Um so merkwürdiger ist der Haufen um

$$
\alpha=2^{\mathrm{h}}{ }^{\circ} \mathrm{O}^{\mathrm{m}} \quad \delta=+5^{\circ} \cdot 4 \quad(\mathrm{I} 855.0)
$$

wo sich sehr viele Nebel auf engem Raum zusammenfinden. Es sind zumeist recht schwache Objekte, zentral verdichtet und etwas flockiger Form, so daß sie den Eindruck ganz ferner Sternhäufchen machen. Ein langes Band größerer Nebelhäufigkeit zieht in dieser Gegend von Norden nach Süden. und die angegebene Stelle bildet den dichtesten Teil desselben.

Astrophysikalisches Institut Königstuhl-Heidelberg, I 909 Juni.

Max Wolf.

Inhalt zu Nr. 4352. W. Miinch. Photometrische Beobachtungen veränderlicher Sterne. 113. $-W$. F. Denning. Heights of meteoric fireballs. 131. - M. Wolf. Über einen Nebelhaufen in Cetus. I3I. 